\title{
Impact of Renewable Energy Forecast Imperfections on Market-Clearing Outcomes
}

\author{
Ordoudis, Christos; Pinson, Pierre
}

Published in:

Proceedings of IEEE International Energy Conference 2016

Link to article, DOI:

10.1109/ENERGYCON.2016.7513962

Publication date:

2016

Document Version

Peer reviewed version

Link back to DTU Orbit

Citation $(A P A)$ :

Ordoudis, C., \& Pinson, P. (2016). Impact of Renewable Energy Forecast Imperfections on Market-Clearing Outcomes. In Proceedings of IEEE International Energy Conference 2016 IEEE.

https://doi.org/10.1109/ENERGYCON.2016.7513962

\section{General rights}

Copyright and moral rights for the publications made accessible in the public portal are retained by the authors and/or other copyright owners and it is a condition of accessing publications that users recognise and abide by the legal requirements associated with these rights.

- Users may download and print one copy of any publication from the public portal for the purpose of private study or research.

- You may not further distribute the material or use it for any profit-making activity or commercial gain

- You may freely distribute the URL identifying the publication in the public portal

If you believe that this document breaches copyright please contact us providing details, and we will remove access to the work immediately and investigate your claim. 


\title{
Impact of Renewable Energy Forecast Imperfections on Market-Clearing Outcomes
}

\author{
Christos Ordoudis and Pierre Pinson \\ Department of Electrical Engineering \\ Technical University of Denmark \\ Kgs. Lyngby, Denmark \\ \{chror, ppin\}@dtu.dk
}

\begin{abstract}
The increased integration of renewable energy sources, in particular wind and solar power, calls for changes in power system operation. Current market designs that are only efficient to accommodate limited uncertainty are highly challenged by the partly predictable renewable energy generation. Hence, innovative market structures have been proposed to cope with the uncertainty introduced. Nonetheless, the quality of wind power forecasts may affect the market outcome due to their inaccuracy. For this reason, a framework is proposed to examine market-clearing algorithms, both deterministic and stochastic approaches, under imperfect wind power forecasts in order to quantify their influence on the market outcome. Results show that mean value mismatch between "estimated" and "realized" distributions has the highest impact on total system cost. Finally, it is examined if cost recovery for market players is guaranteed in the presence of inaccurate wind power forecasts.
\end{abstract}

Index Terms-Bilevel programming, electricity markets, renewable energy forecasting, stochastic programming.

\section{INTRODUCTION}

Current electricity market mechanisms in Europe are based on a sequential clearing of two trading floors. The dayahead market, which is cleared 12-36 hours before real-time operation and the balancing market, which deals with actual imbalances that arise during the real-time operation. Moreover, stochastic producers (e.g., wind and solar) participate in the market with a very low marginal cost which gives them priority over conventional generators, as the day-ahead market is cleared based on the least-cost merit-order principle. As the actual production of renewable energy sources is not known in advance, forecasts are needed in order to schedule the units at the day-ahead stage. However, the production of renewables is characterized by stochasticity and non-dispatchability. Hence, current market designs are highly challenged and novel ones that cope with the inherent renewable uncertainty have to be considered [1]. New market-clearing approaches often assume that access to the correct description of the stochastic process pertaining to renewable energy generation is available. In practice, this may not hold true, and thus their robustness under imperfect renewable energy forecasts needs to be evaluated.

As the penetration of renewable energy increases in power systems, having accurate forecasts is of high importance. The work in [2] showed that operational advantages, system cost savings and reduced wind curtailment are some of the benefits

978-1-4673-8463-6/16/\$31.00 (c) 2016 IEEE of using accurate wind power forecasts for the day-ahead scheduling. Authors in [3] examined which statistical measures of wind forecast error affect the solution quality of a unitcommitment problem and to what degree it is affected by the inaccuracy of the statistical information. It was concluded that variance has the highest impact on the results among variance, skewness and kurtosis, pointing out the need to study the impact of the accuracy of each statistical measure independently. Modeling conditional forecast error for wind farms in different locations is presented in [4] by taking into account spatial correlations and how it improves generation scheduling. These studies reveal the need to study the accuracy of statistical measures and spatial correlation, when issuing scenarios for renewable energy in systems with high shares of renewables. A model showing how to incorporate the forecast error of renewable sources of energy in an economic dispatch problem is presented in [5], calculating the probability distribution function of power production and system operating cost. Moreover, the study of [6] models power offers as probabilistic estimates and assesses the impact of imperfect information on a pool market, showing the importance of better rewarding forecast quality in a probabilistic framework.

The purpose of this paper is to propose an evaluation framework for market-clearing mechanisms under imperfect forecasts of stochastic producers. We place ourselves in a probabilistic setting and investigate the effect of stochastic power production mis-estimation on the market outcome. In this context, the proposed framework models imperfect forecasts and evaluates their impact on the outcomes of various market-clearing algorithms. The proposed model is applied on a case study where stochastic producers consist of various wind power generators. However, these could be any other sources of uncertain power production. The power outputs of wind farms are characterized using Beta distributions and are spatially correlated with a correlation factor $\rho$, while the forecast inaccuracy is simulated by utilizing two different distributions that characterize wind power at the day-ahead and real-time stage. The distributions are differentiated in terms of their mean value and variance, while the spatial correlation factor is also differentiated to examine how each of them affects the market outcome. It is shown that the mismatch of the mean value between the "estimated" and "realized" distributions highly affects total system cost, whereas the mis- 
match of variance and spatial correlation have a lower impact. As stated in [7], flexible producers recover their cost for any realization of stochastic production in the conventional dispatch and improved dispatch of stochastic producers, whereas in stochastic dispatch cost recovery of flexible producers is only guaranteed in expectation and not for any realization of uncertainty. This property holds if stochastic production is accurately described with a proper scenario set $\Omega$. Hence, we investigate if cost recovery is guaranteed for conventional and stochastic producers. Results show that under stochastic dispatch, cost recovery is not guaranteed for some balancing generators even in expectation, in case of significant mismatch between the "estimated" and "realized" distributions.

The rest of the paper is organized as follows: In Section II three market-clearing designs to be evaluated are described. Section III presents the evaluation framework, while the application results are presented in Section IV. Finally, conclusions and future work are given in Section V.

\section{MARKet-Clearing Mechanisms}

In this study, we examine three market-clearing approaches, extensively presented in [7], against wind power forecast imperfections. We follow the market settlement scheme described in [7], which is an energy-only market. For the reader's convenience, the same setup and notations as in [7] are used hereby. The conventional dispatch, which is a sequential clearing of day-ahead and balancing markets, is modeled. Moreover, the stochastic dispatch that co-optimizes the day-ahead and balancing markets, and the improved dispatch, which additionally enforces the least-cost merit-order principle, are examined. We focus on a single time period, as no intertemporal constraints, e.g., ramping limits are considered in the formulation. Furthermore, network constraints are included in the model, while the demand is assumed to be completely inelastic. Two trading floors are considered in all marketclearing setups. Namely, the first stage is the day-ahead market and the second one is the balancing market, which deals with deviations from the day-ahead schedule. Two types of power generators are considered in problem formulation, a set of $I$ conventional generators and a set of $J$ stochastic generators.

\section{A. Conventional dispatch (ConvD)}

The sequential market clears each market independently by solving optimization models (1) and (2). The total cost of the day-ahead dispatch is minimized by optimization model (1):

$$
\begin{array}{ll}
\underset{\Theta^{\mathrm{DA}}}{\operatorname{Min}} & C^{\mathrm{DA}}\left(p_{i}, p_{j}\right) \\
\text { s.t. } & h^{\mathrm{DA}}\left(p_{i}, p_{j}, \delta^{0}\right)-L=0: \lambda^{\mathrm{DA}}, \\
& g^{\mathrm{DA}}\left(p_{i}, \delta^{0}\right) \leq 0, \\
& p_{j} \leq \widehat{W},
\end{array}
$$

where $\Theta^{\mathrm{DA}}=\left\{p_{i}, p_{j}, \delta^{0}\right\}$ is the set of optimization variables of the day-ahead dispatch problem. The optimal day-ahead dispatch $\left(p_{i}^{*}, p_{j}^{*}\right)$ of conventional and stochastic producers is determined by model (1). The optimal dispatch provided by the model follows the least-cost merit-order principle, i.e., clearing the market based on ascending order of energy production marginal prices. Additionally, vector $\delta^{0}$ stands for the voltage angles and $L$ for the load at each node of the power system. The vector $\lambda^{\mathrm{DA}}$ of dual variables of the power balance constraint (1b) represents the locational marginal prices. Constraints (1b)-(1c) describe the equalities and inequalities that formulate the day-ahead market-clearing problem. The upper bound of stochastic generators is enforced by (1d) and is equal to the expected generation $\widehat{W}$. The balancing market model (2) has to deal with the imbalances introduced by stochastic producers by having the day-ahead schedule $\left(p_{i}^{*}, p_{j}^{*}\right)$ fixed. A vector $y_{\omega^{\prime}}$ is introduced that includes the decision variables of the balancing market that account for energy imbalances. The actions that can take place during the real-time operation of the system and comprise vector $y_{\omega^{\prime}}$ are up/down power regulation of flexible units, spillage of stochastic production and shedding of load.

Model (2) minimizes the cost of balancing energy and identifies the optimal balancing actions $y_{\omega^{\prime}}^{*}$, as follows:

$$
\begin{array}{ll}
\underset{\Theta^{\mathrm{B}}}{\operatorname{Min}} & C^{\mathrm{B}}\left(y_{\omega^{\prime}}\right) \\
\text { s.t. } & h^{\mathrm{B}}\left(y_{\omega^{\prime}}, \delta_{\omega^{\prime}}, \delta^{0 *}\right)+W_{\omega^{\prime}}-p_{j}^{*}=0: \lambda_{\omega^{\prime}}^{\mathrm{B}}, \\
& g^{\mathrm{B}}\left(y_{\omega^{\prime}}, \delta_{\omega^{\prime}}, p_{i}^{*} ; W_{\omega^{\prime}}\right) \leq 0,
\end{array}
$$

where $\Theta^{\mathrm{B}}=\left\{y_{\omega^{\prime}}, \delta_{\omega^{\prime}}\right\}$ is the set of optimization variables of the balancing market problem. Vectors $\delta_{\omega^{\prime}}$ and $\lambda_{\omega^{\prime}}^{\mathrm{B}}$ denote the voltage angles and the locational marginal prices at each node of the system at the balancing stage. Constraints (2b)-(2c) describe the equalities and inequalities that formulate the balancing market-clearing problem. The total cost of operation is equal to $C^{\mathrm{DA}}\left(p_{i}^{*}, p_{j}^{*}\right)+C^{\mathrm{B}}\left(y_{\omega^{\prime}}^{*}\right)$ for the specific realization $W_{\omega^{\prime}}$ of stochastic production.

\section{B. Stochastic dispatch (StochD)}

Stochastic integrated market jointly clears the day-ahead and balancing markets and is formulated as the two-stage stochastic programming problem (3). The day-ahead market is represented at the first stage and the balancing market at the second one. The following model aims at minimizing the total expected operating cost and is extensively described in [8]. Model (3) writes as follows:

$$
\begin{array}{cl}
\underset{\Theta^{\mathrm{ST}}}{\operatorname{Min}} & C^{\mathrm{DA}}\left(p_{i}, p_{j}\right)+\mathbb{E}_{\omega}\left[C^{\mathrm{B}}\left(y_{\omega}\right)\right] \\
\text { s.t. } & h^{\mathrm{DA}}\left(p_{i}, p_{j}, \delta^{0}\right)-L=0: \lambda^{\mathrm{DA}}, \\
& g^{\mathrm{DA}}\left(p_{i}, \delta^{0}\right) \leq 0, \\
& p_{j} \leq \bar{W}, \\
& h^{\mathrm{B}}\left(y_{\omega}, \delta_{\omega}, \delta^{0}\right)+W_{\omega}-p_{j}=0, \quad \forall \omega \in \Omega, \\
& g^{\mathrm{B}}\left(y_{\omega}, \delta_{\omega}, p_{i} ; W_{\omega}\right) \leq 0, \quad \forall \omega \in \Omega,
\end{array}
$$

where $\Theta^{\mathrm{ST}}=\left\{p_{i}, p_{j}, \delta^{0} ; y_{\omega}, \delta_{\omega}, \forall \omega\right\}$ is the set of optimization variables of the stochastic integrated market. The stochastic production is modeled by a finite set of scenarios $\Omega$. Additionally, it is described by a vector $W_{\omega}$ and a probability of realization $\pi_{\omega}$, where $\sum_{\omega \in \Omega} \pi_{\omega}=1$. Capacities of stochastic power production are described by a vector $\bar{W}$ and the expectation 
operator is defined by $\mathbb{E}_{\omega}$ over the scenario set $\Omega$ weighted by the probability $\pi_{\omega}$. In this formulation, stochastic production is constrained by the installed capacity $\bar{W}$, instead of the expected generation $\widehat{W}$ that was used in model (1). Moreover, day-ahead scheduling is not fixed, meaning that the dayahead decisions enter the problem as variables. Constraints (3e)-(3f) are included in the model for all scenarios $\omega \in \Omega$ and an expected balancing cost is introduced in the objective function (3a). These model specifications allow the interaction between the two stages and anticipate the balancing actions that occur in the real-time operation of the power system. Flexible units may be dispatched out of merit-order to help the system deal with all the imbalances in real-time operation.

\section{Improved dispatch of stochastic producers (ImpD)}

A stochastic model that provides an improved dispatch of stochastic producers is proposed in [7]. It is formulated as a bilevel programming problem, where the upper-level problem (4a)-(4d) minimizes the total expected operating cost and is constrained by the lower-level problem (4e)-(4h). The latter is equivalent to the conventional day-ahead dispatch (Model (1)) that enforces the least-cost merit-order principle. The only difference lies on the computation of the upper bound of stochastic production $\left(p_{j}^{\max }\right)$, where in the case of model (4) is defined by the upper-level problem and included in the lower-level problem, having an impact on generation scheduling and power flows of the day-ahead stage. Additionally, lower-level variables affect the total expected cost of the upperlevel problem. As a result, model (4) enforces the least-cost merit-order principle to conventional producers and also takes into account the cost of uncertainty of the stochastic producers. The bilevel programming problem writes as follows:

$$
\begin{array}{cl}
\underset{\Theta^{\mathrm{IDU}}}{\operatorname{Min}} & C^{\mathrm{DA}}\left(p_{i}, p_{j}\right)+\mathbb{E}_{\omega}\left[C^{\mathrm{B}}\left(y_{\omega}\right)\right] \\
\text { s.t. } & h^{\mathrm{B}}\left(y_{\omega}, \delta_{\omega}, \delta^{0}\right)+W_{\omega}-p_{j}=0, \quad \forall \omega \in \Omega, \\
& g^{\mathrm{B}}\left(y_{\omega}, \delta_{\omega}, p_{i} ; W_{\omega}\right) \leq 0, \quad \forall \omega \in \Omega, \\
& 0 \leq p_{j}^{\max } \leq \bar{W}, \\
& \left(p_{i}, p_{j}, \delta^{0}\right) \in \arg \left\{\underset{\Theta^{\mathrm{IDL}}}{\operatorname{Min}} . C^{\mathrm{DA}}\left(x_{i}, x_{j}\right)\right. \\
\text { s.t. } & h^{\mathrm{DA}}\left(x_{i}, x_{j}, \theta^{0}\right)-L=0: \lambda^{\mathrm{DA}}, \\
& g^{\mathrm{DA}}\left(x_{i}, \theta^{0}\right) \leq 0, \\
& \left.x_{j} \leq p_{j}^{\max }\right\},
\end{array}
$$

where $\Theta^{\mathrm{IDU}}=\left\{p_{i}, p_{j}, \delta^{0}, p_{j}^{\max } ; y_{\omega}, \delta_{\omega}, \forall \omega\right\}$ and $\Theta^{\mathrm{IDL}}=$ $\left\{x_{i}, x_{j}, \theta^{0}\right\}$ are the sets of optimization variables of the upper- and lower-level problems, respectively. The bilevel problem (4) is transformed into a single level problem by replacing the lower-level problem (4e)-(4h) by its KarushKuhn-Tucker (KKT) conditions, since it is linear. Then it is transformed to a Mixed-Integer Linear Program (MILP) problem in order to handle the bilinear terms that arise from the complementarity conditions.

\section{EVALUATION FrAMEWORK}

The robustness of the market-clearing algorithms, presented in Section II, is evaluated based on the total system cost, unit dispatch, wind spillage, load shedding and market players' profits. Stochastic power production is modeled by a finite scenario set $\Omega$. The scenario sets are derived from different distributions, which are used to characterize stochastic power production at day-ahead and real-time stage in order to simulate inaccurate forecasts. The distributions are differentiated in terms of their mean value and variance. Additionally, the spatial correlation factor of the scenarios issued is differentiated to examine how it affects the market outcome.

Let us consider $J$ stochastic generators in different locations. Two different distributions are utilized to model the mismatch of the scenarios used for the day-ahead stage and the real-time stage of the problem. A "realized" distribution $G^{*}$ defined by a set of parameters $\theta^{*}$ is constructed in order to sample $\Omega_{G}$ realization scenarios of stochastic production. Moreover, an "estimated" distribution $\hat{F}$ defined by a set of parameters $\hat{\theta}$ is used to sample $\Omega_{F}$ scenarios for the dayahead stage of clearing the market. Stochastic production is equal to $y \bar{W}$, where $y \in[0,1]$ is the realization of the random variable $Y$ following either $Y \sim G^{*}\left(y ; \theta^{*}\right)$ or $Y \sim \hat{F}(y ; \hat{\theta})$. Parameters $\theta^{*}$ and $\hat{\theta}$ are defined by the mean value and variance, i.e., $\left(\mu^{*}, \sigma^{2 *}\right)$ and $\left(\hat{\mu}, \hat{\sigma}^{2}\right)$, respectively. Moreover, the scenarios describing stochastic generation in different locations are modeled by a Gaussian copula, as described in [9]. A correlation factor $\rho_{j^{\prime}, j^{\prime \prime}}$ is used to determine the spatial correlation between stochastic generators $j^{\prime}$ and $j^{\prime \prime}$.

Market-clearing algorithms base the day-ahead schedule on forecasts of stochastic production. The "estimated" distribution may not model accurately the actual output of stochastic production, given the limited predictability of renewable power generation. We model forecast imperfections by introducing a mis-estimation parameter $\epsilon$, which is common for all $J$ producers. The three mis-estimation cases are shown below:

- Mismatch of the mean value: $\left(\hat{\mu}_{j}, \hat{\sigma}_{j}^{2}\right)=\left(\epsilon \cdot \mu_{j}^{*}, \sigma_{j}^{2 *}\right)$, given $\hat{\rho}_{j^{\prime}, j^{\prime \prime}}=\rho_{j^{\prime}, j^{\prime \prime}}^{*}$.

- Mismatch of the variance value: $\left(\hat{\mu}_{j}, \hat{\sigma}_{j}^{2}\right)=\left(\mu_{j}^{*}, \epsilon \cdot \sigma_{j}^{2 *}\right)$, given $\hat{\rho}_{j^{\prime}, j^{\prime \prime}}=\rho_{j^{\prime}, j^{\prime \prime}}^{*}$.

- Mismatch of the spatial correlation factor: $\hat{\rho}_{j^{\prime}, j^{\prime \prime}}=$ $\epsilon \cdot \rho_{j^{\prime}, j^{\prime \prime}}^{*}$, given $\left(\hat{\mu}_{j}, \hat{\sigma}_{j}^{2}\right)=\left(\mu_{j}^{*}, \sigma_{j}^{2 *}\right)$.

In our simulations, we consider only wind power producers as the stochastic power generation. In line with existing literature, e.g. [10], wind power production is modeled using a Beta distribution $B(\alpha, \beta)$. Parameters $\alpha$ and $\beta$ are calculated based on $\left(\mu, \sigma^{2}\right)$ values by:

$$
\alpha=\left(\frac{1-\mu}{\sigma^{2}}-\frac{1}{\mu}\right) \mu^{2}, \quad \beta=\alpha\left(\frac{1}{\mu}-1\right) .
$$

Firstly, models (1), (3) and (4) are solved having as input $\Omega_{F}$ equiprobable scenarios for wind power. The optimal dayahead dispatch $\left(p_{i}^{*}, p_{j}^{*}\right)$ is derived along with the voltage angles $\delta^{0 *}$ that define the power flows in the power system. Additionally, the day-ahead cost of operating the system $C^{\mathrm{DA}}\left(p_{i}^{*}, p_{j}^{*}\right)$ is calculated. The real-time operation of the market is modeled by having $\Omega_{G}$ equiprobable realizations of wind power production. Model (2), which describes the balancing market, is solved independently for each wind 
power realization on the basis of an "out-of-sample analysis". Optimal balancing actions $y_{\omega_{G}}^{*}$ are derived for each wind realization and the balancing cost $C^{B}\left(y_{\omega_{G}}^{*}\right)$ is calculated. The real-time operating cost $C^{R T}$ is calculated based on $C^{R T}=$ $\sum_{\omega_{G} \in \Omega_{G}} \pi_{\omega_{G}} C^{B}\left(y_{\omega_{G}}^{*}\right)$. Total operating cost of the system $T C$ is given by $T C=C^{\mathrm{DA}}\left(p_{i}^{*}, p_{j}^{*}\right)+C^{R T}$. Furthermore, locational marginal prices of day-ahead and balancing stages are calculated. Having the aforementioned values, the profits of conventional and stochastic producers can be calculated along with individual costs for each balancing action (i.e, up/down regulation, wind spillage and load shedding costs). Details on calculation of operating costs and profits can be found in [7].

\section{Application Results}

The proposed evaluation framework presented in Section III is tested on the single-area version of the IEEE Reliability Test System [11]. The characteristics of the power system used are based on [12]. Data that are modified with respect to [12] are provided in Table I. In our case, the number of generators is increased to provide a smoother supply curve. Up and down regulation costs are equal to 1.1 and 0.91 of the energy offer price $\left(C_{i}\right)$. Maximum up/down reserve offered $\left(R_{i}^{+}, R_{i}^{-}\right)$ by each unit is based on [7]. The total system demand is equal to $2500 \mathrm{MW}$. In order to introduce congestions in the transmission system, capacities of transmission lines were modified. Specifically, the capacities of lines 1-5, 5-10 and $7-8$ are increased to $350 \mathrm{MW}$, whereas in the case of lines $13-23,14-16$ and $15-21$ are reduced to $250 \mathrm{MW}, 325 \mathrm{MW}$ and $500 \mathrm{MW}$, respectively. Two wind farms are located at nodes 5 and 7 of the power system and the capacity of each wind farm is equal to $450 \mathrm{MW}$. Wind spillage action is considered cost free, while load shedding cost is equal to $\$ 1000 / \mathrm{MWh}$. In order to evaluate the robustness of market-clearing designs under wind power forecast imperfections, the parameters of the "realized" distribution $G^{*}$ are chosen $\left(\mu_{j}^{*}, \sigma_{j}^{2 *}\right)=(0.55,0.05)$ and $\rho_{j^{\prime}, j^{\prime \prime}}^{*}=0.35$. Imperfect forecasts are simulated by changing the mis-estimation parameter $\epsilon$, which takes values in interval [0.6:0.1:1.4], and the "estimated" correlation factor $\hat{\rho}_{j^{\prime}, j^{\prime \prime}}$, which takes values in interval [-1:0.25:1]. The aforementioned intervals are chosen to represent in a realistic way forecast inaccuracy. We use 1000 wind power scenarios for the day-ahead stage based on the "estimated" distribution and 1000 wind power realizations sampled from the "realized" distribution for the simulations.

\section{A. Total system operating cost}

Initially, the three market-clearing mechanisms are compared in terms of the operating system cost. Fig. 1, 2 and 3 illustrate how the system cost is affected when the mean value, variance and spatial correlation factor are mismatched. It can be observed in Fig. 1 that the minimum value of system cost is attained when the parameter $\epsilon$ is equal to 1 . In this case, there is no mismatch of the mean value. However, in cases of underestimation or overestimation, the system cost increases for all the three market-clearing algorithms. This is more intense in cases of overestimation due to the increased
TABLE I

COnVEnTIONAL Unit DATA

\begin{tabular}{cccccc}
\hline Unit \# & $\begin{array}{c}P_{i}^{\max } \\
(\mathrm{MW})\end{array}$ & $\begin{array}{c}R_{i}^{+} \\
(\mathrm{MW})\end{array}$ & $\begin{array}{c}R_{i}^{-} \\
(\mathrm{MW})\end{array}$ & $\begin{array}{c}C_{i} \\
(\$ / \mathrm{MWh})\end{array}$ & Bus ID \\
\hline 1 & 152 & 40 & 40 & 18.24 & 1 \\
2 & 40 & 30 & 30 & 27 & 1 \\
3 & 152 & 40 & 40 & 13.24 & 2 \\
4 & 40 & 30 & 30 & 28 & 2 \\
5 & 300 & 70 & 70 & 23.70 & 7 \\
6 & 197 & 60 & 60 & 20.47 & 13 \\
7 & 197 & 60 & 60 & 21.47 & 13 \\
8 & 197 & 60 & 60 & 22.47 & 13 \\
9 & 60 & 60 & 60 & 25.95 & 15 \\
10 & 155 & 30 & 30 & 14.42 & 15 \\
11 & 155 & 30 & 30 & 12.42 & 16 \\
12 & 400 & 0 & 0 & 7.23 & 18 \\
13 & 400 & 0 & 0 & 7.24 & 21 \\
14 & 300 & 0 & 0 & 5.46 & 22 \\
15 & 155 & 30 & 30 & 10.42 & 23 \\
16 & 155 & 30 & 30 & 11.42 & 23 \\
17 & 350 & 40 & 40 & 11.70 & 23 \\
\hline
\end{tabular}

up-regulation cost and costly load shedding. More specifically, it can be observed that overestimating the mean value by $40 \%$ results in a higher cost of $11.7 \%, 6 \%$ and $2.5 \%$ for ConvD, ImpD and StochD, respectively. On the other hand, underestimating the mean value by $40 \%$ causes a system cost increase of $3.2 \%, 1.8 \%$ and $1.7 \%$ for ConvD, ImpD and StochD, respectively.

The comparison of ConvD and ImpD shows that they react in similar way for all the values of parameter $\epsilon$, while ImpD always achieves a lower system operating cost. ConvD schedules the expected wind power generation $\widehat{W}$ at the dayahead stage in each case, whereas ImpD schedules wind in a more sophisticated manner. In cases of underestimation, more wind is scheduled with $\operatorname{ImpD}$ compared to ConvD, resulting in a decreased day-ahead cost, which then does not lead to a highly increased real-time cost, as more wind is realized. On the contrary, in cases of overestimation, less wind is scheduled at the day-ahead stage by ImpD compared to ConvD, which protects the system against costly load shedding during extremely low wind power realizations.

Additionally, StochD achieves the lowest system cost in all cases. The system cost obtained is less affected by the underestimation and overestimation of wind power production. Less wind power is scheduled at the day-ahead stage by StochD and significantly less load shedding occurs. The dayahead scheduling of conventional and wind power producers does not follow the least-cost merit-order principle, while it anticipates the cost of balancing actions. Even though the system cost is affected by the mismatch of the mean value, there is enough flexibility scheduled in the system to cope with significantly inaccurate forecasts.

Results in Fig. 2 show the total system cost for imperfect wind power forecasts when the variance is mismatched. System cost is not affected in cases of ImpD and ConvD, while StochD is more sensitive to the scenario structure. The minimum system cost is achieved for accurate wind power forecasts (i.e., $\epsilon=1$ ). Moreover, ConvD provides the most expensive running of the system, while StochD provides the lowest system cost for the whole interval of parameter $\epsilon$. 


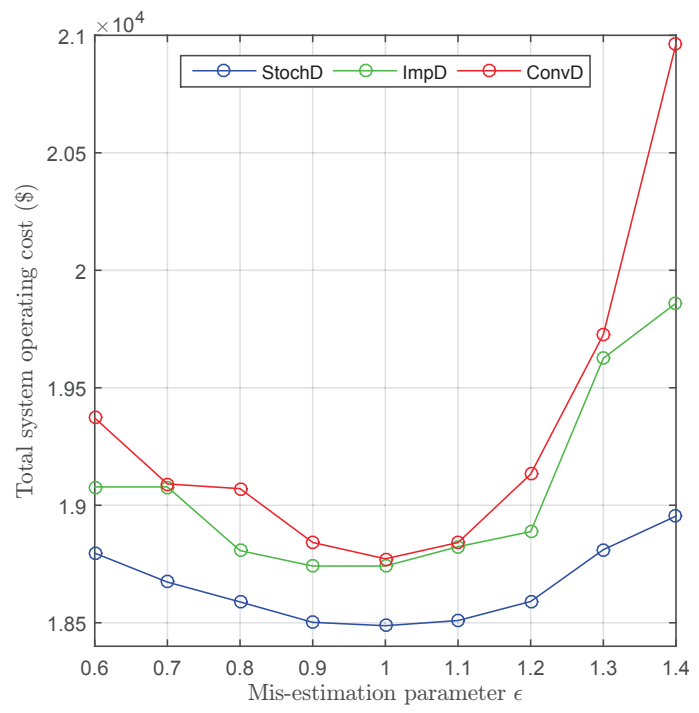

Fig. 1. Mismatch of mean value. Total system operating cost as a function of mis-estimation parameter $\epsilon$.

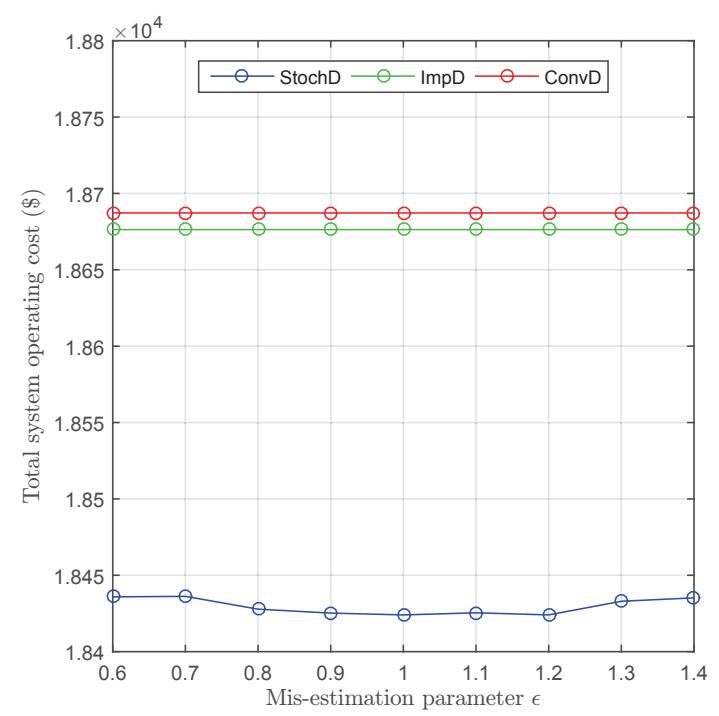

Fig. 2. Mismatch of variance value. Total system operating cost as a function of mis-estimation parameter $\epsilon$.

Similar conclusions can be drawn in case of mismatching spatial correlation factor $\rho$, as illustrated in Fig. 3. The vertical line shows $\rho_{j^{\prime}, j^{\prime \prime}}^{*}=0.35$. The system cost is not affected in the case of ConvD, while it slightly drops between $\hat{\rho}_{j^{\prime}, j^{\prime \prime}}=-1$ and $\hat{\rho}_{j^{\prime}, j^{\prime \prime}}=-0.5$, when the market is cleared based on ImpD. Also in this case, StochD results in the lowest system cost and shows a sensitivity on the mismatch of spatial correlation factor. The system cost is decreasing as "estimated" $\hat{\rho}_{j^{\prime}, j^{\prime \prime}}$ is picked more accurately by being closer to $\rho_{j^{\prime}, j^{\prime \prime}}^{*}=0.35$. In these sets of simulations, no load shedding occurs for any of the market-clearing algorithms.

\section{B. Expected profits}

As described in Section I, cost recovery for producers is guaranteed only in expectation for StochD. In order to preserve the theoretical properties of StochD described in [8]

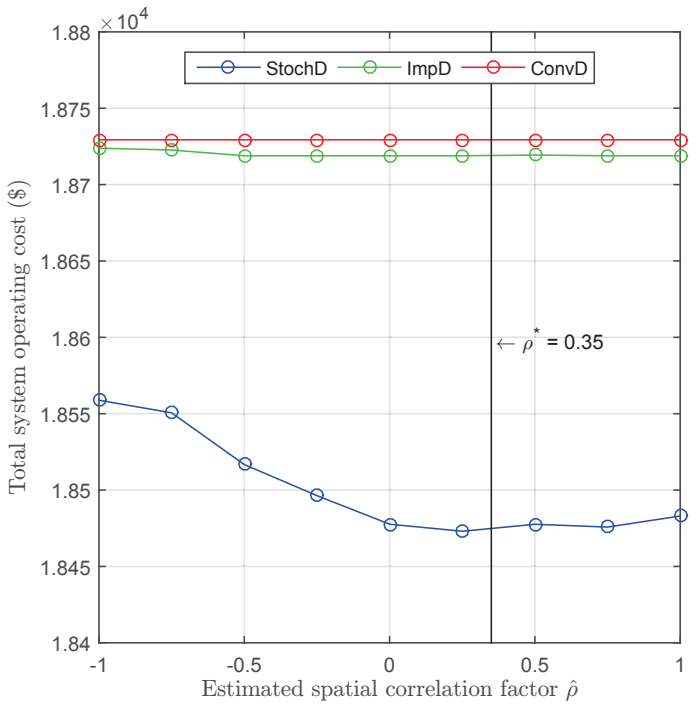

Fig. 3. Mismatch of spatial correlation factor. Total system operating cost as a function of estimated spatial correlation factor $\hat{\rho}$.

(i.e., revenue reconciliation and cost recovery in expectation), modeling of the uncertain wind power production has to be accurate enough. However, cost recovery of conventional power producers is not guaranteed in expectation, as shown in Fig. 4, when the mean value is overestimated. It can be observed that conventional Unit 2 is facing negative profit in expectation when $\epsilon>1$. This shows that even a $10 \%$ overestimation of the mean value will result most probably to losses for this producer. The lower day-ahead prices compared to balancing prices along with the usual usage of Unit 2 for down-regulation results in this phenomenon. The aforementioned situation was mostly observed for flexible units that were scheduled out of merit-order to provide down-regulation. For the cases of ConvD and ImpD, no negative profits in expectation were noticed. Unit 2 is not scheduled at the day-ahead stage, so it only provides up-regulation in real-time operation. This results in positive profit in expectation, as Unit 2 is not dispatched in a loss-making position in the day-ahead market.

Fig. 5 illustrates the expected profit of a wind power producer, owning both wind farms. In this case, it can be noted that StochD better protects the producers that bring uncertainty into the system, as the expected profit is positive in all cases of mismatching the mean value. However, this is not the case for ConvD and ImpD. The costly action of load shedding results in high balancing prices, when the mean value is overestimated and finally low wind production is realized. In this case, wind power producer faces great losses that lead to negative profit in expectation.

The optimization problems have been solved using CPLEX 12.6 under GAMS. The simulations were performed on a stationary computer with Intel i7 4-core processor clocking at $3.4 \mathrm{GHz}$ and $8 \mathrm{~GB}$ of RAM. The average total time of solving, for the whole mis-estimation interval, the day-ahead market and the balancing market problem for 1000 different wind power scenarios and realizations was 1380 seconds for ConvD, 1000 seconds for StochD and 9140 seconds for ImpD. 


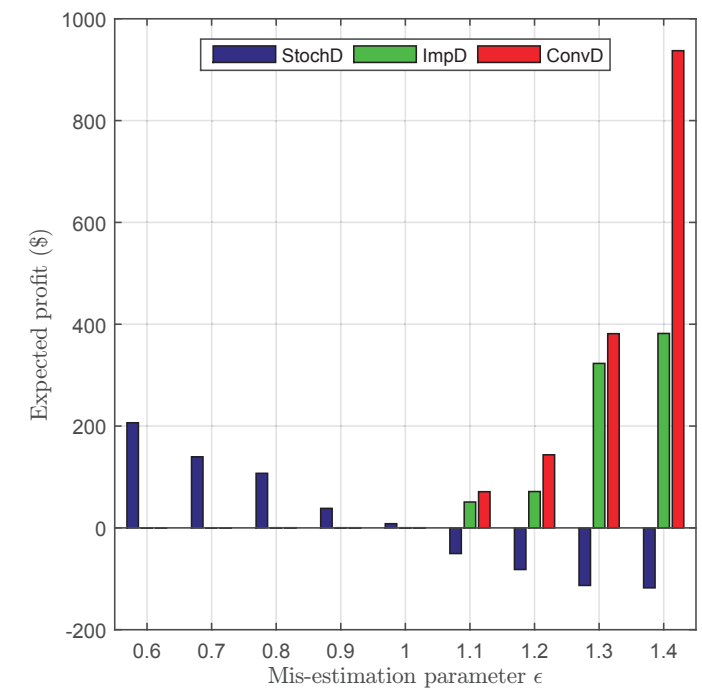

Fig. 4. Mismatch of mean value. Expected profit of conventional Unit 2 as a function of mis-estimation parameter $\epsilon$.

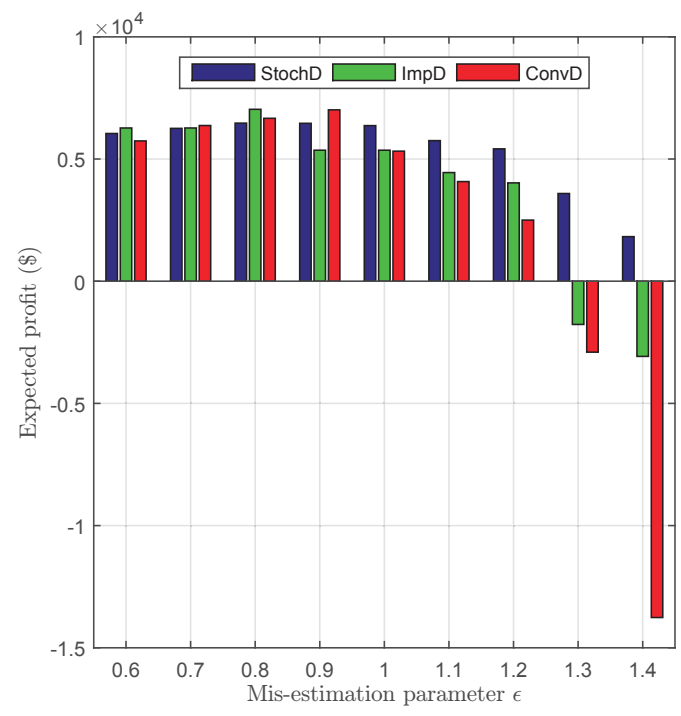

Fig. 5. Mismatch of mean value. Expected profit of wind power producer as a function of mis-estimation parameter $\epsilon$.

\section{CONClusions AND Future Work}

This paper offers a framework to evaluate the robustness of market-clearing algorithms under forecast imperfections. The robustness is evaluated based on system cost and generation scheduling, along with a discussion on the expected profits of conventional and stochastic producers. In our simulations, stochastic generators were wind farms and the power output was characterized by Beta distribution. Moreover, a factor $\rho$ was used to capture the spatial correlation of the wind farms. Forecast imperfections were modeled by using two different distributions for the "estimated" and "realized" wind power production. The distributions were differentiated in terms of mean value and variance. Additionally, a mismatch of spatial correlation factor was simulated. Results show that the mismatch of the mean value highly affects the system cost compared to mismatching variance or spatial correlation factor. Stochastic dispatch (StochD) provided the lowest system cost in all cases, while conventional dispatch (ConvD) the highest one. Only the market outcome of StochD was affected when having inaccurate forecasts in terms of variance and spatial correlation factor. Regarding the expected profits, flexible conventional producers may face negative profit in cases of overestimating the mean value under StochD market-clearing algorithm. Furthermore, wind power producer encounters negative profit in expectation, when implementing ConvD and improved dispatch of stochastic producers (ImpD), mainly due to the high balancing prices in case of load shedding. For future research, we will introduce different mis-estimation parameters for stochastic producers. This will give a better insight on how the market outcome is affected, as it will allow us to model various degrees of mis-estimation depending on the wind farm location. Finally, further studies within our framework will allow providing relevant feedback for improvements in market design in a probabilistic framework.

\section{ACKNOWLEDGEMENTS}

The work of the authors is partly funded by the Danish Strategic Research Council (DSF) through projects "5s-Future Electricity Markets", No. 12-132636/DSF and "CITIES", No. 1305-00027B/DSF.

\section{REFERENCES}

[1] P. Pinson and M. O'Malley, "Foreword for the special section on wind and solar energy: Uncovering and accommodating their impacts on electricity markets," IEEE Trans. Power Syst., vol. 30, no. 3, pp. 15571559, 2015.

[2] E. Mc Garrigle and P. Leahy, "Quantifying the value of improved wind energy forecasts in a pool-based electricity market," Renew. Energy, vol. 80, pp. 517-524, 2015.

[3] C. Lowery and M. O'Malley, "Impact of wind forecast error statistics upon unit commitment," IEEE Trans. Sustain. Energy, vol. 3, no. 4, pp. 760-768, 2012.

[4] N. Zhang, C. Kang, Q. Xia, and J. Liang, "Modeling conditional forecast error for wind power in generation scheduling," IEEE Trans. Power Syst., vol. 29, no. 3, pp. 1316-1324, 2014.

[5] G. J. Osorio, J. M. Lujano-Rojas, J. C. O. Matias, and J. P. S. Catalao, "Including forecasting error of renewable generation on the optimal load dispatch," in Proc. IEEE PowerTech, 2015, pp. 1-6.

[6] A. Papakonstantinou and P. Pinson, "Towards electricity markets accommodating uncertain offers," 13th Int. Work. Large-Scale Integr. Wind Power Transm. Networks, 2014.

[7] J. M. Morales, M. Zugno, S. Pineda, and P. Pinson, "Electricity market clearing with improved scheduling of stochastic production," Eur. J. Oper. Res., vol. 235, no. 3, pp. 765-774, 2014.

[8] J. M. Morales, A. J. Conejo, and K. Liu, "Pricing electricity in pools with wind producers," IEEE Trans. Power Syst., vol. 27, no. 3, pp. 13661376, 2012.

[9] S. Delikaraoglou and P. Pinson, "High-quality wind power scenario forecasts for decision-making under uncertainty in power systems," in 13th Int. Work. Large-Scale Integr. Wind Power Transm. Networks, 2014.

[10] A. Fabbri, T. G. San Román, J. R. Abbad, and V. H. M. Méndez Quezada, "Assessment of the cost associated with wind generation prediction errors in a liberalized electricity market," IEEE Trans. Power Syst., vol. 20, no. 3, pp. 1440-1446, 2005.

[11] C. Grigg et al., "The IEEE Reliability Test System-1996. A report prepared by the Reliability Test System Task Force of the Application of Probability Methods Subcommittee," IEEE Trans. Power Syst., vol. 14, no. 3, pp. 1010-1020, 1999.

[12] A. J. Conejo, M. Carrión, and J. M. Morales, Decision Making Under Uncertainty in Electricity Markets. New York: Springer, 2010, vol. 153. 